\title{
Safety of the Neurological and Brain Activities from Oxidative Stress and Metabolic Syndrome Associated with Obesity
}

\section{Kamal A. Amin ${ }^{1,2 *}$}

${ }^{1}$ Department of Biochemistry, Beni Suef University, Egypt

${ }^{2}$ Department of Biochemistry, El Dammam University, Saudi Arabia

Nowadays there are many diet compositions and beverages, juice and industrial progress provide man with huge desire for eating and drinking without real control.

Few researches concerned with diet disturbances include high fat diet (HFD) high sucrose diet, (HSD), and food additives on the body organs as liver kidney and heart. Some of them include insulin resistances, metabolic syndrome and associated disorders of fatty liver, renal pathology, and cardiovascular disturbances.

The number of people with diabetes worldwide will increase from 171 million in 2000 to 366 million by 2030 with a noticeable increase from the developing countries. These numbers are affected by oxidative stress associated with renal, hepatic and cardiovascular complication with different scale, age, nutrition and life style.

Adipose tissue has long been considered as a mere energy store but is increasingly appreciated for its roles in participating in several physiological and biochemical events such as inflammation, angiogenesis, hypertension, and vascular homeostasis. Also, adipose tissue synthesizes adipokines (adiponectin, leptin, resistin, angiotensinogen and TNF- $\alpha$ ) which characterized for their role in influencing energy homeostasis, feeding behavior and may play a role in mediating long-term potentiation, neuroprotection and neuroinflammation.

Failure of adipocytes to remove FFA from the circulation and deposition of adipose/lipid into muscle, liver, or bone marrow is known to promote not only metabolic syndrome but localized tissue dysfunction and a variety of health complications including atherosclerosis, and ultimately the development of metabolic syndrome. Excess generation of mitochondrial ROS due to hyperglycemia initiates a vicious circle by activating stress-sensitive pathways.

Enhanced production of AGEs, sorbitol and proinflammatory cytokines exerts a positive feedback on ROS and RNS synthesis and potentiates PKC-mediated vascular dysfunction by altering gene expression as well as vascular function and structure [1].

\section{Do We have a Problem with Neurological Disturbances with High Fat Eating?}

Few manuscripts reported that the metabolic changes may represent a pathological bridge between metabolic syndrome (obesity) and neurological disorders such as stroke, cognition, Alzheimer's disease and depression. Others demonstrated that the type of diet affects the brain structure and function.

Consumption of fat-rich food is one of the most important factors that may lead to increase in the prevalence and risk of cardiovascular and neurological diseases [2,3]. Saturated fat, hydrogenated fat and cholesterol can profoundly impair memory and hippocampal morphology [4].

Recently, Ryan et al. [5] reported that excess lipid flux into the central nervous system leads to activation of nutrient-sensing pathways in the hypothalamus, including PKC, PPAR $\gamma$, ER stress, and inflammatory mediators. We still need an inclusive answer on the diet, brain and neurological disorders.

Our hypothesis is that high fat diet associated with obesity induced inflammation will be associated with increased levels of proinflammatory mediators (PGE2, TNF- $\alpha$, IL-1 $\beta$ ) leading, in turn, to disturbances in neurotransmitters and brain antioxidant-oxidative stress ratio. The diet overeating induce obesity that produce oxidative stress. It stimulates inflammation-neurotransmitters axis (obesityoxidative stress- inflammation-neurotansmittors) which may help to explain the link between obesity and the increased risk of nervous disorders in obese and aged persons.

We are still in need for some experimental and clinical evidences that links neurological disorders and diet disturbances especially high fat diets. Plan of lifestyle, diet and pharmacological handling that interrupt the obesity-inflammation-neurotransmitter axis may be useful for reducing the risk of neurological disorders or its progression. We suggest that these oxidative stress, inflammatory and neurotransmitters pathways are potential novel pathophysiological and pharmacological targets for the management of obesity-associated neurodisorders.

\section{References}

1. Johansen JS, Harris AK, Rychly DJ, Ergul A (2005) Oxidative stress and the use of antioxidants in diabetes: linking basic science to clinical practice. Cardiovasc Diabetol 4: 5.

2. Amin KA, Kamel HH, Abd Eltawab MA (2011) The relation of high fat diet metabolic disturbances and brain oxidative dysfunction: modulation by hydroxy citric acid. Lipids Health Dis 10: 74

3. Namavar MR, Raminfard S, Jahromi ZV, Azari H (2012) Effects of high-fat diet on the numerical density and number of neuronal cells and the volume of the mouse hypothalamus: a stereological study. Anat Cell Biol 45: 178-184.

4. Granholm AC, Bimonte-Nelson HA, Moore AB, Nelson ME, Freeman LR, et al. (2008) Effects of a saturated fat and high cholesterol diet on memory and hippocampal morphology in the middle-aged rat. J Alzheimers Dis 14: 133-145.

5. Ryan KK, Woods SC, Seeley RJ (2012) Central nervous system mechanisms linking the consumption of palatable high-fat diets to the defense of greater adiposity. Cell Metab 15: 137-149.

${ }^{*}$ Corresponding author: Kamal A. Amin, Department of Biochemistry, El Dammam University, Saudi Arabia, E-mail: kaamin10@yahoo.com

Received January 25, 2013; Accepted January 28, 2013; Published January 30 2013

Citation: Amin KA (2013) Safety of the Neurological and Brain Activities from Oxidative Stress and Metabolic Syndrome Associated with Obesity. Biochem Anal Biochem 2:e133. doi:10.4172/2161-1009.1000e133

Copyright: (C) 2013 Amin KA. This is an open-access article distributed under the terms of the Creative Commons Attribution License, which permits unrestricted use, distribution, and reproduction in any medium, provided the original author and source are credited. 